\title{
Natural Product Chemistry Research in Iraq
}

\author{
A. A. Al-Jeboory*1. Y. Z. FARID ${ }^{2}$ \\ ${ }^{1 \& 2}$ College of pharmacy, Uruk University. Baghdad. Iraq. \\ alialjeboory@yahoo.com
}

\begin{abstract}
Today's west an east scientists developed synthetic drugs but because of problems of the side effects and high cost in addition to resistant developed which not happened in using natural drugs this push the scientist in all over the world to look for new drugs from natural compounds. In Iraq. Since 1980 up today we started a plan led by scientist in Chemistry, Pharmacology, Physician, Biology in all universities $\&$ medical research centers and we started to develop a new method for extraction, isolation, ident cation of active constituent and trying the active constituent biologically and pharmacologically in invitro and in vivo on experimental animals. We started screening of more than thousand plants which indigenous in Iraq and take the knowledge from folkloric physician and old information about these from ancient books and references we found around two hundred promising plants we developed methods of extraction, isolation using chromatography and trying to evaluate their efficacy. We managed to prepare drugs to treat warts from Ficus Caria vitexin from Crataegus amorous, prostrating anew antihyperlipidemic drug from prosopis fractal etc. A new method of isolation and identification by new phytochemical methods and new experimental tool for each active constituent are as example how to separate alkaloids, cardiac glycosides inotropic agents, polyphenoles etc. In addition to bacteriological, toxi-pathological methods then pass to prepare anew natural drugs prepared with highly sophisticated methods and with highly efficiency which mav be better than svnthetic drugs and more safe .
\end{abstract}

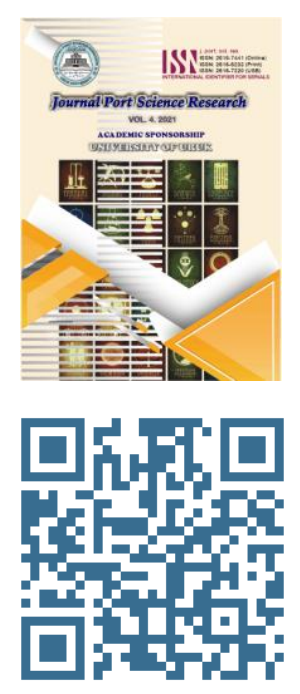

Crossref

$10.36371 /$ port.2021.1.8

Keywords: natural compounds, antihyperlipidemic drug, bacteriological ,toxi-pathological.

\section{INTRODUCTION}

Plant based medicaments for centuries means prime therapeutic weapon, are still in the front line today for treating a large number of diseases. Furthermore, the deeper we go into the study of plants used in traditional medicine, the more we see new possibilities for additional plant derivatives. Plants represent today, and will represent in the future, a first class source for new medicaments both in industrialized and developing countries. Medical ( or those parts of medicinal plants that we call "crude drugs" are the raw material with to prepare the following derivatives :

1) Decoctions and infusion.

2) Total extracts and tinctures prepared in accordance with the pharmacopeias.

3) Purified and standardized extracts.

4) Chemically pure active products.

Each of them represents a step in the passage from traditional to modern medicine but each of them has its own value and function, and not necessary the chemically pure products are the most active forms of a plant. Generally

speaking therapeutic agents obtained from plant kingdom are considered to be less toxic as compared to those of synthetic origin. Virtually every pharmacological class of drug includes a natural product prototype that exhibits effects of the pharmacological category in question, most of them are plant derived

Table 1 : some of above drugs are plants origin (5)

\begin{tabular}{|l|l|}
\hline Cardio tonic & - cardiac glycosides ( Ianatosides, etc.) \\
\hline Analgesic & - alkaloids ( morphine, codeine, etc.) \\
\hline Antihypertensive & - alkaloids ( reserpine, etc.) \\
\hline Antimalarial & - alkaloids ( quinine, etc.) \\
\hline Cathartic & - anthraquinones ( sennosides, etc.) \\
\hline
\end{tabular}

There are more than 50 categories of secondary organic constituents known from the world of higher plants. The prospects of investigations of plants are Promising, since it has been estimated that only $5-6 \%$ of the worlds flora (approximately 600000 plants )less than $10 \%$ of organic constituents are known, that fully $90 \%$ remains for discovery and investigation. The importance of screening little-studied plants species for the presence of substances having potential medicinal significance is widely understood. This type of work has gained even more importance because lately who is keenly interested in development and utilization of medicinal plant resources step in the search of ingredients from plant:

Al-Jeboory, A. and Farid, Y., 2021. Natural Product Chemistry Research in Iraq. Journal port Science Research, 4(1), pp 41-45. https://doi.org/10.36371/port.2021.1.8 
1) Collection, selection and identification of plant species

2) Preparation of plant extracts

3) Preliminary biological screening

4) Isolation and characterization of plant constituents

5) Structural elucidation (chemical synthesis of analogues)

6) Pharmacological testing

7) Standardization

From this list it is obvious that medicinal plant research is a truly interdisciplinary field.

Basically there are two ways of looking for new biologically active compounds, we can either search for the effect what plants or plant product produce (biological/pharmacological screening ), or we search for certain compounds (phytochemical screening ). As the biological screen is concerned both therapeutic and toxic effects are equally interesting at this stage of the investigation.

In biological screening the work is directed by bioassay, which means that the first question the researcher asks : "dose this or that plant contain any substance with a biological and /or toxicological effect?"

In case of phytochemical screening we are looking for certain type of constituents, therefore the question is :"does this or that plant contain e.g. alkaloids, saponins , etc.?"

\section{Why natural products drug?}

Example of the use of a herbal preparation in modern medicine is the foxglove plant. This herb has been in use since 1775 for the treatment of heart failure. Other examples Salicylic acid, a precursor of aspirin, which was originally derived from white willow bark and the meadow sweet. Cinchona bark is the source of malaria- fighting quinine. Vincristine Is used to treat certain types of cancer and comes from periwinkle.

Beside their use and advantages as a source for drugs, many plants are toxic. There are reports about cases of serious adverse events after administration of herbal products. For instance, several woman developed rapidly progressive interstitial renal fibrosis after taking Chinese herbs which were prescribed by a sliming clinic. Several herbal products interact with conventional drugs such as Echinacea with anabolic steroids, garlic with warfare and Liquorice with Spironlactone. These plant-derived drug and other, which are in use nowadays and still are drugs of choice for special diseases, are preferred on synthetic drugs for the following reasons:

1) Have less side effects and toxicity than synthetic drugs.

2) There is less resistance from microorganisms against these drugs.

3) Can be obtained from cheap resources.
4) They are pure drugs of natural origin.

Alkaloids, leurocristine and vincaleucoblastine, isolated from the Madagascan periwinkle catharnthus roseus (Vinca rosea ) are present in a very small concentrations among the about 60 different monomeric and dimeric indole and oxindole alkaloids. To isolate about $10 \mathrm{~g}$ of leurocristine, 5 tons of dried leaves have been extracted.

\section{Extraction}

The extraction and purification of plant constituents is mainly carried out using simple solid /liquid extraction and a combination of liquid /liquid extraction .

Solid/liquid extraction is diffusion, and it take place at least between two phases. Extraction can be characterized with diffusion and high in cell soap and in the solvent at the beginning of the operation. Both diffuses are based on the fick low, which states:

$\mathrm{dG}=\mathrm{D} . \mathrm{F} . \mathrm{dc} / 1 . \mathrm{t}, \quad$ where $: \mathrm{F}=$ surface $\mathrm{dc}=$ conceration difference $t=$ time $l=$ length of diffusion processes $D=$ diffusion constant

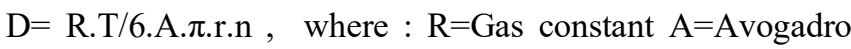
number $\mathrm{R}=$ radius of particulars Temperature $\mathrm{T}=$ temperature

Liquid/liquid extraction are based on the Nerst distribution low: $K_{d}=c 1 / c 2$. Which simply states that the ratio of the concentration in one of the phase to that in the other is constant at a constant temperature .

$\mathrm{C}_{1}$ being the concentration of compound in one of the phase

$\mathrm{C}_{2}$ the concentration in the other phase, and

$\mathrm{K}_{\mathrm{d}}$ is the distribution (or partition ) coefficient

Extraction curves

1. Plant material. 2. Extract

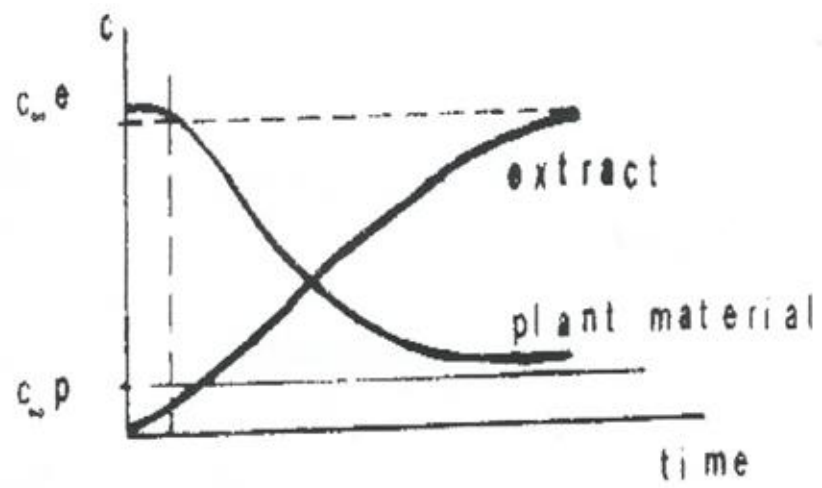

(h)

\section{Figure (1) shows the plant material and the extract}

The precise mode of extraction naturally depends on the texture and water content of the plant material being extracted 
and on the type of substance that is being isolated .In general it is necessary to "kill" the plant tissue (in case of fresh material , to prevent hydrolyses , enzymic oxidation, ect.) Before extraction. From fresh material alcohol, in any case is a good solvent for extraction. (Maceration, blender extraction, etc.) With exhaustive extraction all the low

Pharmacological activity tests
A. LD50 measurements of extract and isolated constituent .
B. Pharmacological effect on
C. Isolated tissue in Krebs:
D. Isolated right and left paced atria.
E. Isolated guinea pig ileum and rabbit jejunum .
F. Isolated cat paced papillary muscle.

\section{2 -In vivo}

Using anaesthetized cat, rabbit, dog, rat and guinea pig measuring the following parameters :
A. blood pressure
B. $\mathrm{dp} / \mathrm{dt}$
C. skeletal muscle tone of solos type in cats also here the extract and the isolated active constituent are used.

\section{Parameters used to elucidate structure of Hypericin as example :}
1) U.V.
2) I.R .
3) Melting point
4) N.M.R
5) Pharmacological results and toxical effects compared with synthetic Hypericin. [5]

\section{New culture techniques}

Another approach to circumventing also growth rate in plantcell suspension culture is to transfer the plant genes which code for the enzymes catalyzing the desired biosynthetic reactions, in to a bacterial or fungal cell. This could lower fermentation times to 24 to 28 hours instead of the 6 to 8 weeks necessary for fermentation with a typical plant- cell suspension. Many gene produce are required to produce desired secondary metabolites. The technology to accomplished this is not yet available, but one-or-two-step enzyme-alyzed conversions should be possible now. More complicated reaction sequences will soon follow .

In the future, successful commercial production of plant drug by cell-culture techniques will depend on the development of strategies that increase yields and shorten fermentation times. The manipulations of plant cells, plant genes, enzymes, rather than of the plants them-selves, should provide us with a host of useful medicinal agents .

Types of New Natural Drug
Just what kind of new drugs will scientists be attempting to find in nature or to cultivate in this manner in the 21 century? Diseases for which satisfactory cures still remain to be developed include :

1) Viral diseases, such as herpes ( genitalis, simplex, and zoster), AIDS,, and certain cancers;

2) Diseases of unknown etiology, including arthritis, some cancers, muscular dystrophy, and Parkinsonism;

3) Self -inflicted diseases, such as alcoholism, drug dependency ,obesity smoking, and stress ;

4) Genetic diseases, ranging from cystic fibrosis and hemophilia to sickle- cell disease .

In addition, improved drugs need to be developed for the control of symptoms such as pain. A nonaddicting narcotic is, for example, urgently needed. Also required are treatments for conditions not readily positioned in any one of the above categories, for instance, elevated cholesterol levels, hypertension. The main natural drug sources include:

1) Carbohydrates and related compounds . Compounds composed of carbon ,hydrogen , and oxygen as polyhydroxy aldehyde or ketone alcohols: sucrose , lactose,stach ,acacia ,tragacanth, agar ,pectin.

2) Glycosides . Substances that on hydrolysis yields one or more sugars among the products of the reaction : barbalion, glycyrrhizin, vanillin,salicon, amygdalin. Also include are Tannins, a group of complex phenolic compounds capable of combining with proteins : hamamelitannin, gallotannic acid.

3) Lipids .Compounds comprising fixed oils, fact, and waxes. Fixed oils and fact. Glyceryl esters of fatty acids that are saponified by alkalies: olive oil ,peanut oil ,castor oil. Waxes esters of fatty acids with high -molecular -weight monohdydric alcohols :beeswax, spermaceti, carnauba wax.

4) Volatile oil. Essential oils that represent the odoriferous principles of plants :peppermint oil,clove oil, cinnamon oil , anise oil, rose oil.

5) Resin and resin combinations . Compounds comprising resins, oleoresins, oleo-gum-resins , and balsamsa. Resins. Solid or semisolids amorphous product of complex chemical nature : resin , podophyllum resin, jalap resin. Oleoresins .Resins and volatile oils in homogeneous mixtures:turpentine , copaiba. Oleo-gunresins.oleorsins and gums in homogeneous mixtures: turpentine, myrrh . Balsams . Resins with mixtures of aromatic substances such as benzoic acid,cinnamic acid, or both :benzoic tolu balsam ,perubalsam ,styrax.

6) Steroids. Derivatives of cyclopenten-ophenanthrene : estrogens adrenal, cortex hormones ,cardio active aglycones, bid, cholesterol,ergosterol. 
7) Alkaloids .Nitrogenous crystalline or oily compounds, usually basic in character atropine ,morphine, quinine, cocaine ,reserpine.

8) Peptide hormones .Active principles secreted by certain endocrine glands: glucagon ,insulin ,oxytocin vasopressin , ACTH.

9) Enzymes and other protein ,Organic catalysts product by living organism :pepsin ,pancereatin , rennin ,papain ,trypsin Nitrogenous organic substances composed of amino acids units :gelatin, Heparin ,protamine .

10) Vitamins .Chemical compounds necessary for normal growth and function of animals :thiamine ,riboflavin ,cyanocobalamin ,ascorbic acid, tocopherol.

11) Antibiotic. Chemical entities product biosynthetically that are destructive or inhibitory to microorganisms : penicillin ,cephalosporin, Polymxin ,tetracycline, erythromycin , kanamycin griseodulvin, gentamicin.etc

\section{Essential\& seldom Elements}

Some clinical studies that $\mathrm{Zn}$ therapy can be beneficial in some cases of atherosclerosis. Zinc sulphate given orally to patients with advanced vascular disease for 29 months, showed marked improvement in $12 / 13$ cases. In another study, 16 patients 914 with improvement symptomatic atherosclerosis and 2 who had disabling vasopastic disease ) were given zinc sulphate for 3-11 months. Six of these were excluded because of change in their diet or habits. Clinical improvement observed in 6 of the remaining 10 was ascribed to $\mathrm{Zn}$ therapy. Four experienced no improvement; showed rapid progression of their disease.

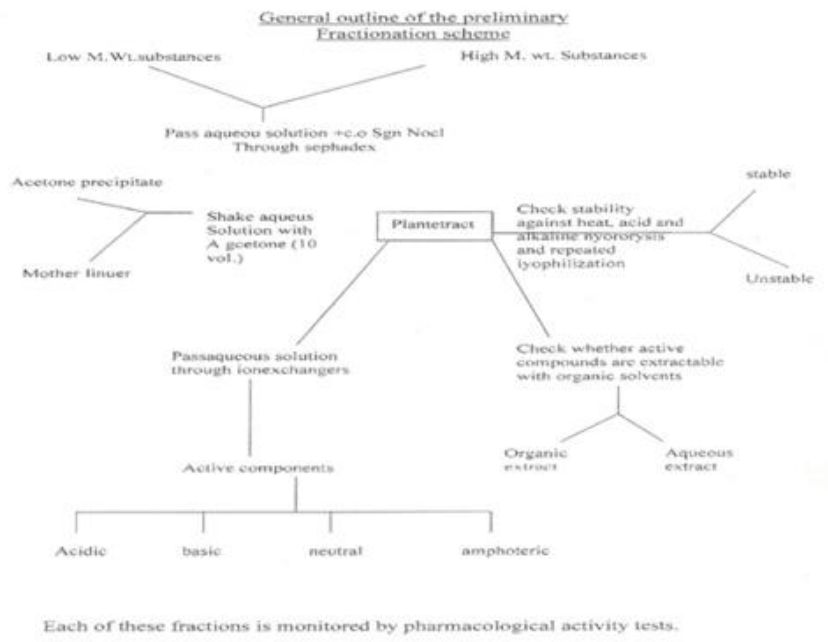

Figure 2 shows general outline of the preliminary fractionation scheme [6]
12) Bilogics . Product composed of antigenic matter or antibody preparations capable of developing a state of immunity in the patient: Adsorbed diphtheria toxoid ,hepatitis B vaccine, poliomyelitis vaccines, Immune globulins, diphtheria antitoxin, also biologics related to human blood :albumin human , antihemohilic factor.

13) Allergens. Substances ,usually protoplasmic in origin that cause unusual responses in hypersensitive individuals :pollen grains ,mold spores, feathers, animal dander, poison ivy.

14) Poisonus plant .Higher plants and fungi that produce toxic effects when introduced into the human body :jimson weed, nightshade, water hemlock. Amanita ,inocybe.

15) Herbs and "health foods" products of natural origin used by Folk medicine in the self-treatment of disease state or less -than-optimal health conditions many are without therapeutic effect and some are toxic $[5,4]$.

While the available information is still fragmentary and grossly inadequate for any meaningful application, one fact emerges beyond any grossly shadow of doubt that elements elicit significant effect on physiopathology of the heart. There are definite to the links between:

a. $\mathrm{Cr}, \mathrm{Cu}, \mathrm{Li}, \mathrm{Mg}, \mathrm{Si}, \mathrm{V}, \mathrm{Zn}$, etc. and blood cholesterol levels and atherosclerosis :

b. $\mathrm{Ba}, \mathrm{Cl}, \mathrm{Cu}, \mathrm{Ge}, \mathrm{Hg}, \mathrm{I}, \mathrm{Pb}$, etc. (in general ) $\mathrm{Cd}$ and $\mathrm{Na}$ (in particular) and blood pressure disturbances :

a. (c) $\mathrm{Al}, \mathrm{Cu}, \mathrm{Fe}, \mathrm{Mn}, \mathrm{Ni}, \mathrm{Zn}$, etc., and myocardial infraction:

b. (d) $\mathrm{Cu}, \mathrm{Mg}, \mathrm{D}, \mathrm{Se}, \mathrm{Zn}$, etc. and throboembolism and coronary heart disease;

c. (e) $\mathrm{Ba}, \mathrm{Ca}, \mathrm{Hg}, \mathrm{K}, \mathrm{Li}, \mathrm{Mg}$, etc. and cardiac arrhythmia;

d. (f) $\mathrm{Hg}, \mathrm{Na}, \mathrm{O}$, etc. and congestive heart failure.

Many other elements influence the cardiac function due to their direct or indirect ( through interaction with other elements ) actions in physiological or toxic concentions. Some other like, I, Kr and $\mathrm{Xe}$ (asradiosiotopes ) find diagnostic uses in the measurement of cardiac output and pulmonary circulation abnormalities. The trace element selenium, cadmium act as antioxidant to be used as antihyperlipidemic drugs. 


\section{REFERENCES}

[1] Pharmacognosy Workbook, by Ralph Bienfang, Associate Professor of Pharmacognosy, University of Oklahoma. Ninth Edition, revised. Published by the Transcript press, Norman, Oklahoma. (1938). The Journal of the American Pharmaceutical Association (1912), 27(12), 1283. https://doi.org/10.1002/jps.3080271233

[2] Barisione, G., Baroffio, M., Crimi, E., \& Brusasco, V. (2010). Beta-adrenergic agonists. Pharmaceuticals. MDPI AG. https://doi.org/10.3390/ph3041016

[3] Burnstock, G., Dobson, J., Liang, B., \& Linden, J. (1998). Cardiovascular Biology of Purines (pp. 342-358). Boston, MA: Springer US.

[4] DiBianco, R., Shabetai, R., Kostuk, W., Moran, J., Schlant, R. C., \& Wright, R. (1989). A Comparison of Oral Milrinone, Digoxin, and Their Combination in the Treatment of Patients with Chronic Heart Failure. New England Journal of Medicine, 320(11), 677-683. https://doi.org/10.1056/nejm198903163201101

[5] Giebisch, G., Klein-Robbenhaar, G., Klein-Robbenhaar, J., Ratheiser, K., \& Unwin, R. (1993). Renal and extrarenal sites of action of diuretics. Cardiovascular Drugs and Therapy, 7(1 Supplement), 11-21. https://doi.org/10.1007/BF00877954

[6] Stanton, B. A. (1990). Cellular actions of thiazide diuretics in the distal tubule. Journal of the American Society of Nephrology, 1(5), 832-836. https://doi.org/10.1681/asn.v15832

[7] Townsend, R. R., \& Holland, O. B. (1990). Combination of converting enzyme inhibitor with diuretic for the treatment of hypertension. Archives of Internal Medicine. https://doi.org/10.1001/archinte.150.6.1175 